\title{
A STRONG LAW FOR $B$-VALUED ARRAYS
}

\author{
DELI LI, M. BHASKARA RAO, AND R. J. TOMKINS
}

(Communicated by Richard Durrett)

\begin{abstract}
Let $(B,\|\cdot\|)$ be a real separable Banach space and $\left\{X_{n, k} ; n \geq\right.$ $1,1 \leq k \leq n\}$ a triangular array of iid $B$-valued random variables. Set $S(n)=\sum_{k=1}^{n} X_{n, k}, n \geq 1$, and $\log t=\log \max \{e, t\}, t \in \mathfrak{R}$. In this paper, we characterize the limit behavior of $S(n) / \sqrt{2 n \log n}, n \geq 1$. As an application of our result, we resolve an open problem posed by $\mathrm{Hu}$ and Weber (1992). The case of row-wise independent arrays is also dealt with.
\end{abstract}

\section{INTRODUCTION}

Let $\left\{X, X_{n, k} ; 1 \leq k \leq n, n \geq 1\right\}$ be a triangular array of iid real random variables. Hu, Móricz, and Taylor [4] obtained the Strong Law of Large Numbers by showing that

$$
\frac{1}{n} \sum_{k=1}^{n} X_{n, k}, n \geq 1, \quad \text { converges to } E(X) \text { (finite) a.s. }
$$

if and only if $E X^{2}<\infty$. This means that in order to extend the Strong Law of Large Numbers from sequences to arrays, one has to strengthen the finite first moment condition to the finite second moment.

However, there is a world of difference between sequences of iid random variables and arrays of iid random variables. The Law of the Iterated Logarithm cannot hold for arrays. More precisely, $\mathrm{Hu}$ and Weber [5] proved that

$$
\limsup _{n \rightarrow \infty} \frac{\sum_{k=1}^{n} X_{n, k}}{\sqrt{2 n \log n}}=1 \text { a.s. }
$$

provided

$$
E X=0, E X^{2}=1 \text { and } E X^{4}<\infty,
$$

Received by the editors March 21, 1994.

1991 Mathematics Subject Classification. Primary 60B12, 60F15, 60G50.

Key words and phrases. Almost sure limit, Banach space, cluster set, the Law of the Iterated Logarithm, Strong Law of Large Numbers.

The second author's research was supported in part by a NSF-EPSCoR grant and U.S. Army Research Office Grant DAAH04-93-G-0030.

The third author's research was supported in part by a grant from the Natural Sciences and Engineering Research Council of Canada. 
and that

$$
\limsup _{n \rightarrow \infty} \frac{\sum_{k=1}^{n} X_{n, k}}{\sqrt{2 n \log n}}=\infty \quad \text { a.s. }
$$

provided

$$
E X=0, \quad E X^{2}=1 \text { and } E|X|^{q}=\infty \text { for some } 2<q<4 .
$$

As noted on p. 482 of $\mathrm{Hu}$ and Weber [5], the behavior of $\sum_{k=1}^{n} X_{n, k}, n \geq 1$, when $E|X|^{q}<\infty$ for all $q<4$ but $E X^{4}=\infty$ remains an open problem.

In this paper we characterize precisely the limit behavior of

$$
\sum_{k=1}^{n} X_{n, k} / \sqrt{2 n \log n}, \quad n \geq 1,
$$

thus resolving the open problem posed by $\mathrm{Hu}$ and Weber [5]. Theorem 2.1, the main result of this paper, involves a Banach space setting. In $\S 3$ we consider the case of row-wise independent $B$-valued arrays, generalizing Theorem 2.1.

\section{MAIn Results}

Let us begin with some notation. Let $(B,\|\cdot\|)$ be a real separable Banach space with topological dual $B^{*}$. Let $\left\{X, X_{n, k} ; 1 \leq k \leq n, n \geq 1\right\}$ be an array of iid $B$-valued random variables. Set $S(n)=X_{n, 1}+X_{n, 2}+\cdots+X_{n, n}$, $n \geq 1$. Let $X_{n}, n \geq 1$, be a sequence of iid $B$-valued random variables with the same distribution as $X$. Set $S_{n}=X_{1}+X_{2}+\cdots+X_{n}, n \geq 1$. Let $\log t=\log \max \{e, t\}, t \in \mathfrak{R}$. Let $H$ be the reproducing kernel Hilbert space associated with $\mu=\mathscr{L}(X)$, the law or distribution of $X$, and $K$ the unit ball of $H$. For a study of the properties of $H$ and $K$, see Kuelbs [7]. The symbol $C\left(\left\{Y_{n} ; n \geq 1\right\}\right)$ stands for the cluster set of the family $\left\{Y_{n} ; n \geq 1\right\}$ of random variables. We can now state the main result.

Theorem 2.1. Let $\left\{X, X_{n, k} ; 1 \leq k \leq n, n \geq 1\right\}$ be an array of iid $B$-valued random variables. If

$$
\left\{\begin{array}{l}
E X=0, \\
E\left(\frac{\|X\|^{4}}{(\log \|X\|)^{2}}\right)<\infty, \quad \text { and } \\
\frac{S_{n}}{\sqrt{2 n \log n}}, \quad n \geq 1, \text { converges to } 0 \text { in probability, }
\end{array}\right.
$$

then

$$
\begin{gathered}
P\left(\left\{\frac{S(n)}{\sqrt{2 n \log n}} ; n \geq 1\right\} \text { is conditionally compact in } B\right)=1 \\
C\left(\left\{\frac{S(n)}{\sqrt{2 n \log n}} ; n \geq 1\right\}\right)=K \text { a.s. } \\
\limsup _{n \rightarrow \infty} \frac{\|S(n)\|}{\sqrt{2 n \log n}}=\sup _{x \in K}\|x\| \quad \text { a.s. }
\end{gathered}
$$

Conversely, (2.2) implies (2.1). 
Proof. Note that $K$ is compact if and only if $\left\{f^{2}(X) ; f \in B^{*},\|f\| \leq 1\right\}$ is uniformly integrable. See Kuelbs [7]. Now,

$$
E\left(\frac{\|X\|^{4}}{(\log \|X\|)^{2}}\right)<\infty
$$

implies that $E(\|X\|)^{2}<\infty$. Consequently, $K$ is compact. To show that (2.2) is valid, it will suffice to show that, for each $\varepsilon>0$,

$$
P\left(\frac{S(n)}{\sqrt{2 n \log n}} \in\left(K^{\varepsilon}\right)^{c} \text { i.o. }\right)=0,
$$

where $K^{\varepsilon}=\left\{y \in B ; \inf _{x \in K}\|y-x\|<\varepsilon\right\}$ and $\left(K^{\varepsilon}\right)^{c}=B-\left(K^{\varepsilon}\right)$. Note that

$$
\begin{aligned}
\sum_{n \geq 1} P\left(\frac{S(n)}{\sqrt{2 n \log n}} \in\left(K^{\varepsilon}\right)^{c}\right) & =\sum_{n \geq 1} P\left(\frac{S_{n}}{\sqrt{2 n \log n}} \in\left(K^{\varepsilon}\right)^{c}\right) \\
& =\sum_{n \geq 1} P\left(\inf _{x \in K}\left\|\frac{S_{n}}{\sqrt{2 n \log n}}-x\right\| \geq \varepsilon\right),
\end{aligned}
$$

and that, by Theorem 1.2 of $\mathrm{Li}$ and Wang [11] for $r=2$ (see Theorem 3 of Lai [8] when $B=R$ and $p=4)$,

$$
\left\{\begin{array}{l}
K \text { is compact and for every } \varepsilon>0, \\
\sum_{n \geq 1} P\left(\inf _{x \in K}\left\|\frac{S_{n}}{\sqrt{2 n \log n}}-x\right\| \geq \varepsilon\right)<\infty
\end{array}\right.
$$

if and only if (2.1) holds. (Some comments are in order on the equivalence of (2.1) and (2.7). Li and Wang [11] proved the equivalence of (2.1) and (2.7) by using a result on moderate deviations for partial sums of iid random variables. This result on moderate deviations can be found in Chen [2], de Acosta [3], and Ledoux [9]. When $B$ is the real line, Lai [8] proved the equivalence of (2.1) and (2.7) by using a limit theorem for delayed sums. See Theorem 2 of Lai [8].) Consequently, (2.1) implies that

$$
\sum_{n \geq 1} P\left(\frac{S(n)}{\sqrt{2 n \log n}} \in\left(K^{\varepsilon}\right)^{c}\right)<\infty \text { for every } \varepsilon>0,
$$

and hence, by the Borel-Cantelli Lemma, (2.5) follows and so does (2.2). By some remarks of $\mathrm{Li}$ and Wang ([11], p. 103), (2.1) also implies that, for every $x \in K$,

$$
\sum_{n \geq 1} P\left(\left\|\frac{S_{n}}{\sqrt{2 n \log n}}-x\right\| \leq \varepsilon\right)=\infty \text { for every } \varepsilon>0 .
$$

Thus, for every $x \in K$ and $\varepsilon>0$,

$$
\sum_{n \geq 1} P\left(\left\|\frac{S(n)}{\sqrt{2 n \log n}}-x\right\| \leq \varepsilon\right)=\sum_{n \geq 1} P\left(\left\|\frac{S_{n}}{\sqrt{2 n \log n}}-x\right\| \leq \varepsilon\right)=\infty
$$

Note that

$$
\frac{S(n)}{\sqrt{2 n \log n}}, \quad n \geq 1
$$


is a sequence of independent $B$-valued random variables. By the Borel-Cantelli Lemma, for every $x \in K$,

$$
P\left(\frac{S(n)}{\sqrt{2 n \log n}} \in B(x, \varepsilon) \text { i.o. }\right)=1 \text { for every } \varepsilon>0,
$$

where $B(x, \varepsilon)=\{y \in B ;\|y-x\| \leq \varepsilon\}$. Hence for every $x \in K$,

$$
x \in C\left(\left\{\frac{S(n)}{\sqrt{2 n \log n}} ; n \geq 1\right\}\right) \text { a.s. }
$$

Since $B$ is separable, we get

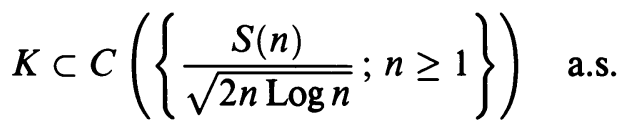

On the other hand, (2.5) implies that

$$
C\left(\left\{\frac{S(n)}{\sqrt{2 n \log n}} ; n \geq 1\right\}\right) \subset K \text { a.s. }
$$

Thus (2.3) follows. Combining (2.2) and (2.3), we get (2.4). Finally, we prove that (2.2) implies (2.1). In fact, the Borel-Cantelli Lemma and (2.2) imply that, for some $0<\Lambda<\infty$,

$$
\sum_{n \geq 1} P\left(\left\|\frac{S(n)}{\sqrt{2 n \log n}}\right\| \geq \Lambda\right)<\infty .
$$

It follows that, for every $\varepsilon>0$,

$$
\sum_{n \geq 1} P\left(\left\|\frac{S_{n}}{n}\right\| \geq \varepsilon\right)<\infty,
$$

and hence that $S_{n} / n, n \geq 1$, converges to 0 a.s. Thus $E X=0$. By a standard argument, one can assume that $X$ is symmetric. By the Lévy inequality and (2.15), we observe that

$$
\sum_{n \geq 1} P\left(\max _{1 \leq k \leq n}\left\|X_{n, k}\right\| \geq \Lambda \sqrt{2 n \log n}\right)<\infty,
$$

which is equivalent to

$$
\sum_{n \geq 1} n P(\|X\| \geq \sqrt{2 n \log n})<\infty .
$$

Thus we get

$$
E\left(\frac{\|X\|^{4}}{(\log \|X\|)^{2}}\right)<\infty .
$$

The convergence in probability of the sequence $S_{n} / \sqrt{2 n \log n}, n \geq 1$, to 0 follows from the facts that

$$
\frac{f(S(n))}{\sqrt{2 n \log n}}=\frac{\sum_{k=1}^{n} f\left(X_{n, k}\right)}{\sqrt{2 n \log n}}, \quad n \geq 1, \text { converges to } 0 \text { in probability }
$$


for every $f \in B^{*}$ and the sequence

$$
\mathscr{L}\left\{\frac{S(n)}{\sqrt{2 n \log n}}\right\}, \quad n \geq 1,
$$

of distributions is tight (since (2.2) holds). Hence (2.2) implies (2.1).

As an application of Theorem 2.1, we solve the open problem proposed by $\mathrm{Hu}$ and Weber [5] in the following way.

Corollary 2.2. Let $\left\{X, X_{n, k} ; 1 \leq k \leq n, n \geq 1\right\}$ be an array of iid real-valued random variables. Then

$$
\begin{gathered}
\limsup _{n \rightarrow \infty} \frac{S(n)}{\sqrt{2 n \log n}}=1 \quad \text { a.s. } \\
\liminf _{n \rightarrow \infty} \frac{S(n)}{\sqrt{2 n \log n}}=-1 \quad \text { a.s. }
\end{gathered}
$$

and

$$
C\left(\left\{\frac{S(n)}{\sqrt{2 n \log n}} ; n \geq 1\right\}\right)=[-1,1] \text { a.s. }
$$

if and only if

$$
E X=0, \quad E X^{2}=1 \text { and } E\left(\frac{X^{4}}{(\log |X|)^{2}}\right)<\infty
$$

\section{SOME FURTHER COMMENTS}

In this section we want to relax the assumption that the array be iid to the assumption that the array be identically distributed and row-wise independent. Such arrays do arise when one is interested in the limit behavior of the sequence of least squares estimators in the environment of linear models. See Kaffes and Bhaskara Rao [6], for example. Tomkins [12] studied the Law of the Iterated Logarithm for double sequences of random variables. Let $\left\{Y, Y_{n, k} ; 1 \leq k \leq\right.$ $n, n \geq 1\}$ be an array of identically distributed and row-wise independent $B$-valued random variables. The limit behavior of the sequence $\sum_{k=1}^{n} Y_{n, k}$, $n \geq 1$, is the main focus of this section. Let $Y_{n}, n \geq 1$, be a sequence of iid $B$-valued random variables with the common distribution the same as that of $Y$. We have the following result.

Theorem 3.1. Let $\left\{Y, Y_{n, k} ; 1 \leq k \leq n, n \geq 1\right\}$ be an array of identically distributed and row-wise independent $B$-valued random variables and $Y_{k}, k \geq$ 1 , a sequence of iid $B$-valued random variables with the common distribution the same as that of $Y$ such that

$$
\left\{\begin{array}{l}
E Y=0, \\
E\left(\frac{\|Y\|^{4}}{(\log \|Y\|)^{2}}\right)<\infty, \quad \text { and } \\
\frac{\sum_{k=1}^{n} Y_{k}}{\sqrt{2 n \log n}}, \quad n \geq 1, \text { converges to } 0 \text { in probability. }
\end{array}\right.
$$


(I) Then

$$
\begin{aligned}
& P\left(\left\{\frac{\sum_{k=1}^{n} Y_{n, k}}{\sqrt{2 n \log n}} ; n \geq 1\right\} \text { is conditionally compact in } B\right)=1, \\
& C\left(\left\{\frac{\sum_{k=1}^{n} Y_{n, k}}{\sqrt{2 n \log n}} ; n \geq 1\right\}\right) \subset K_{Y} \text { a.s. }
\end{aligned}
$$

and

$$
\limsup _{n \rightarrow \infty} \frac{\left\|\sum_{k=1}^{n} Y_{n, k}\right\|}{\sqrt{2 n \log n}} \leq \sup _{x \in K_{Y}}\|x\| \quad \text { a.s. },
$$

where $K_{Y}$ is the unit ball of $H_{Y}$, the reproducing kernel Hilbert space associated with the law $\mathscr{L}(Y)$ of $Y$.

(II) Further, for each given $0 \leq \alpha \leq 1$ and for each $B$-valued random variable $Y$ satisfying (3.1), there exists an array $\left\{Y, Z_{n, k} ; 1 \leq k \leq n, n \geq 1\right\}$ of identically distributed and row-wise independent $B$-valued random variables such that

$$
C\left(\left\{\frac{\sum_{k=1}^{n} Z_{n, k}}{\sqrt{2 n \log n}} ; n \geq 1\right\}\right)=\alpha K_{Y} \quad \text { a.s. }
$$

and

$$
\limsup _{n \rightarrow \infty} \frac{\left\|\sum_{k=1}^{n} Z_{n, k}\right\|}{\sqrt{2 n \log n}}=\alpha\left(\sup _{x \in K_{Y}}\|x\|\right) \text { a.s. }
$$

Proof. The first part of Theorem 3.1 can be proved in much the same way as Theorem 2.1, so we only give the proof of the second part of Theorem 3.1.

Let $Y, Y_{n}, n \geq 1$, be a sequence of iid $B$-valued random variables satisfying (3.1). In all the following cases, let $\left\{Y, X_{n, k} ; 1 \leq k \leq n, n \geq 1\right\}$ be an array of iid $B$-valued random variables.

Suppose $\alpha=0$. Take

$$
Z_{n, k}=X_{k, 1}, \quad 1 \leq k \leq n, n \geq 1 \text {. }
$$

By (3.1),

$$
C\left(\left\{\frac{\sum_{k=1}^{n} Z_{n, k}}{\sqrt{2 n \log n}} ; n \geq 1\right\}\right)=C\left(\left\{\frac{\sum_{k=1}^{n} Y_{k}}{\sqrt{2 n \log n}} ; n \geq 1\right\}\right)=0 \text { a.s., }
$$

in view of the fact that

$$
\lim _{n \rightarrow \infty} \frac{\sum_{k=1}^{n} Y_{k}}{\sqrt{2 n \log n}}=0 \text { in probability, and hence a.s. }
$$

In fact, (3.8) can be deduced from Theorem 1.4 of Berger [1]. Further,

$$
\limsup _{n \rightarrow \infty} \frac{\left\|\sum_{k=1}^{n} Z_{n, k}\right\|}{\sqrt{2 n \log n}}=\limsup _{n \rightarrow \infty} \frac{\left\|\sum_{k=1}^{n} Y_{k}\right\|}{\sqrt{2 n \log n}}=0 \quad \text { a.s. }
$$

Suppose $0<\alpha \leq 1$. Let $\rho=\alpha^{-2} \geq 1$. Observe that

$$
\{1,2,3, \ldots\}=\bigcup_{m \geq 1}\left\{\left[(m-1)^{\rho}\right]+1,\left[(m-1)^{\rho}\right]+2, \ldots,\left[m^{\rho}\right]\right\},
$$


where the sequence of sets indexed by $m$ is pairwise disjoint. Set for each $n \in\left\{\left[(m-1)^{\rho}\right]+1,\left[(m-1)^{\rho}\right]+2, \ldots,\left[m^{\rho}\right]\right\}$ and $m=1,2, \ldots$,

$$
Z_{n, k}=X_{\left[m^{\rho}\right], k}, \quad 1 \leq k \leq n \text {. }
$$

For example, when $\rho=2$, the set array comports itself as follows:

$$
\begin{aligned}
& Z_{1,1} \quad X_{1,1} \\
& Z_{2,1} Z_{2,2} \quad X_{4,1} X_{4,2} \\
& Z_{3,1} Z_{3,2} Z_{3,3} \quad X_{4,1} X_{4,2} X_{4,3}
\end{aligned}
$$

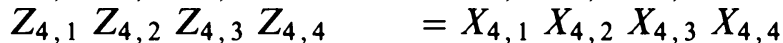

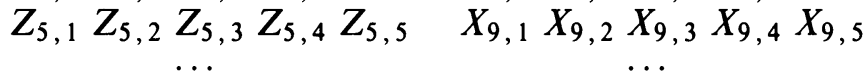

Using the argument presented in Theorem 2.1 with obvious modifications, we have

(3.10)

$$
C\left(\left\{\frac{\sum_{k=1}^{\left[m^{\rho}\right]} Z_{\left[m^{\rho}\right], k}}{\sqrt{2 m^{\rho} \log m}} ; m \geq 1\right\}\right)=C\left(\left\{\frac{\sum_{k=1}^{\left[m^{\rho}\right]} X_{\left[m^{\rho}\right], k}}{\sqrt{2 m^{\rho} \log m}} ; m \geq 1\right\}\right)=K_{Y} \quad \text { a.s. }
$$

Consequently,

$$
\begin{aligned}
& C\left(\left\{\frac{\sum_{k=1}^{n} Z_{n, k}}{\sqrt{2 n \log n}} ; n \geq 1\right\}\right) \supset C\left(\left\{\frac{\sum_{k=1}^{\left[m^{\rho}\right]} Z_{\left[m^{\rho}\right], k}}{\sqrt{2 m^{\rho} \log m^{\rho}}} ; m \geq 1\right\}\right) \\
& =\sqrt{\frac{1}{\rho}} K_{Y}=\alpha K_{Y} \quad \text { a.s. }
\end{aligned}
$$

On the other hand, for each subset $A \subset B$ and $t>0$, by Theorem 3.1 of $\mathrm{Li}$ [10], we have

$$
\begin{aligned}
& P\left(\max _{\left[(m-1)^{\rho}\right]<n \leq\left[m^{\rho}\right]} \inf _{x \in A}\left\|\frac{\sum_{k=1}^{n} Z_{n, k}}{\sqrt{2 m^{\rho} \log m^{\rho}}}-x\right\| \geq t\right) \\
& \quad=P\left(\max _{\left.\left[(m-1)^{\rho}\right]<n \leq\left[m^{\rho}\right]\right]} \inf _{x \in A}\left\|\frac{\sum_{k=1}^{n} X_{\left[m^{\rho}\right], k}}{\sqrt{2 m^{\rho} \log m^{\rho}}}-x\right\| \geq t\right) \\
& \quad \leq 2 P\left(\inf _{x \in A}\left\|\frac{\sum_{k=1}^{\left[m^{\rho}\right]} X_{\left[m^{\rho}\right], k}}{\sqrt{2 m^{\rho} \log m^{\rho}}}-x\right\| \geq t-\mu_{m}\right),
\end{aligned}
$$

where

$$
\mu_{m}=\max _{1 \leq k \leq\left[m^{\rho}\right]} \frac{\operatorname{med}\left\|\sum_{j=k}^{\left[m^{\rho}\right]} X_{\left[m^{\rho}\right], j}\right\|}{\sqrt{2 m^{\rho} \log m^{\rho}}},
$$

which converges to 0 as $m \rightarrow \infty$. (For any random variable $X, \operatorname{med}(\|X\|)$ denotes the median of the real random variable $\|X\|$.) Combining (3.12) and the fact that $m^{\rho} /(m-1)^{\rho}$ converges to unity as $m \rightarrow \infty$, we obtain (3.13)

$$
C\left(\left\{\frac{\sum_{k=1}^{n} Z_{n, k}}{\sqrt{2 n \log n}} ; n \geq 1\right\}\right) \subset C\left(\left\{\frac{\sum_{k=1}^{\left[m^{\rho}\right]} X_{\left[m^{\rho}\right], k}}{\sqrt{2 m^{\rho} \log m^{\rho}}} ; m \geq 1\right\}\right)=\alpha K_{Y} \text { a.s. }
$$

Thus (3.5) holds. (3.6) follows in the same vein. This completes the proof.

Although the Law of the Iterated Logarithm does not hold in general for arrays of row-wise independent random variables, we guess that the following statement is true. 
Conjecture. Let $\left\{Y, Y_{n, k} ; 1 \leq k \leq n, n \geq 1\right\}$ be an array of identically distributed and row-wise independent real-valued random variables. If

$$
E Y=0 \text { and } E Y^{2}=1 \text {, }
$$

then

$$
\limsup _{n \rightarrow \infty} \frac{\sum_{k=1}^{n} Y_{n, k}}{\sqrt{2 n \log \log n}} \geq 1 \text { a.s. }
$$

and

$$
\liminf _{n \rightarrow \infty} \frac{\sum_{k=1}^{n} Y_{n, k}}{\sqrt{2 n \log \log n}} \leq-1 \text { a.s. }
$$

\section{REFERENCES}

1. E. Berger, Majorization, exponential inequalities, and almost sure behavior of vector valued random variables, Ann. Probab. 19 (1991), 1206-1226.

2. X. Chen, Probabilities of moderate deviations for independent random vectors in a Banach space, Chinese J. Appl. Probab. Statist. 7 (1991), 24-32.

3. A. de Acosta, Moderate deviations and associated Laplace approximations for sums of independent random variables, Trans. Amer. Math. Soc. 329 (1992), 357-375.

4. T. C. Hu, F. Móricz, and R. L. Taylor, Strong Laws of Large Numbers for arrays of rowwise independent random variables, Acta Math. Hungar. 54 (1989), 153-162.

5. T. C. Hu and N. C. Weber, On the rate of convergence in the Strong Law of Large Numbers for arrays, Bull. Austral. Math. Soc. 45 (1992), 479-482.

6. D. Kaffes and M. Bhaskara Rao, Weak consistency of least-squares estimators in linear models, J. Multivariate Anal. 12 (1982), 186-198.

7. J. Kuelbs, $A$ strong convergence theorem for Banach space valued random variables, Ann. Probab. 4 (1976), 744-771.

8. T. L. Lai, Limit theorems for delayed sums, Ann. Probab. 2 (1974), 432-440.

9. M. Ledoux, Sur les déviations modérées des sommes de variables aléatoires vectorielles indépendantes de même loi, Ann. Inst. Henri Poincaré 28 (1992), 267-280.

10. D. Li, Convergence rates of law of iterated logarithm for B-valued random variables, Sci. China Ser. A 34 (1991), 395-404.

11. D. $\mathrm{Li}$ and $\mathrm{X}$. C. Wang, Convergence rates for probabilities of moderate deviations for sums of $B$-valued random variables, Proceeding of the Special Year in Probability and Statistics (Tianjin, China, August 1988-May 1989), World Scientific, River Edge, NJ, 1992, pp. $100-110$.

12. R. J. Tomkins, On the law of the iterated logarithm for double sequence of random variables, Z. Wahrsch. Verw. Gebiete 30 (1974), 303-314.

(Deli Li and R. J. Tomkins) Department of Mathematics and Statistics, University of Regina, Saskatchewan, S4S 0A2, Canada

Current address, Deli Li: Department of Mathematics \& Statistics, York University, North York, Ontario, Canada M3J 1P3

(M. Bhaskara Rao) Department of Statistics, North Dakota State university, Fargo, NORTh DAKOTA 58105 